\title{
Charge exchange in the ultraviolet: implication for interacting clouds in the core of NGC 1275
}

\author{
Liyi Gu ${ }^{1}$, Junjie Mao ${ }^{1,2}$, Christopher P. O’Dea ${ }^{3,4}$, Stefi A. Baum ${ }^{3,5}$, Missagh Mehdipour ${ }^{1}$, and Jelle S. Kaastra ${ }^{1,2}$ \\ ${ }^{1}$ SRON Netherlands Institute for Space Research, Sorbonnelaan 2, 3584 CA Utrecht, The Netherlands \\ e-mail: 1.gu@sron.nl \\ 2 Leiden Observatory, Leiden University, PO Box 9513, 2300 RA Leiden, The Netherlands \\ 3 Department of Physics and Astronomy, University of Manitoba, Winnipeg, MB R3T 2N2, Canada \\ 4 School of Physics and Astronomy, Rochester Institute of Technology, Rochester, NY 14623, USA \\ 5 Center for Imaging Science, Rochester Institute of Technology, Rochester, NY 14623, USA
}

Received 10 February 2017 / Accepted 3 March 2017

\begin{abstract}
Context. Charge exchange emission is known to provide a key diagnostic to the interface between hot and cold matter in many astrophysical environments. Most of the recent charge exchange studies focus on its emission in the X-ray band; only a few focus on the UV, even though it can also provide a powerful probe of the charge exchange process.

Aims. An atomic calculation, as well as an application to observed data, are presented to explore and describe the potential use of the UV data for the study of cosmic charge exchange.

Methods. Using the newest charge exchange model in the SPEX code v3.03, we reanalyse the archival Hubble/STIS data of the central region of NGC 1275.

Results. The NGC 1275 spectrum shows hints of three possible weak lines at about $1223.6 \AA$, $1242.4 \AA$, and $1244.0 \AA$, each with a significance of about $2-3 \sigma$. The putative features are best explained by charge exchange between highly ionized hydrogen, neon, and sulfur with neutral matter. The wavelengths of the charge exchange lines are found robustly with uncertainties $\leq 0.3 \AA$. The possible charge exchange emission shows a line-of-sight velocity offset of about $-3400 \mathrm{~km} \mathrm{~s}^{-1}$ with respect to the NGC 1275 nucleus, which resembles a previously reported Ly $\alpha$ absorber. This indicates that the charge exchange lines might be emitted at the same position as the absorber, which could be ascribed to outflowing gas from the nucleus.
\end{abstract}

Key words. atomic processes - galaxies: Seyfert - ultraviolet: general - X-rays: individuals: NGC 1275

\section{Introduction}

Charge exchange $(\mathrm{CX})$ is a key physical process occurring at the interface of cold and hot cosmic plasma. It is well known to produce signature line emission in the X-rays, which provide an important diagnostic of the interaction at the interface. The CX X-rays were first observed in comets as they interact with the solar wind (Lisse et al. 1996; Cravens 1997). Follow-up studies also revealed CX X-rays from the geocoronal and heliospheric areas, and from planets in the solar system (Snowden et al. 2004; Dennerl et al. 2006; Fujimoto et al. 2007; Branduardi-Raymont et al. 2007; Smith et al. 2014). Recently, CX has also been considered as a potential mechanism for X-rays from stellar winds (Pollock 2007), supernova remnants (Lallement 2009; Katsuda et al. 2011), starburst galaxies (Tsuru et al. 2007; Liu et al. 2011), and even galaxy clusters (Fabian et al. 2011; Gu et al. 2015; Hitomi Collaboration et al. 2016b). Most applications of CX to extrasolar X-ray sources are still speculative since the CX X-ray lines from these objects are often found to be rather weak.

The study of CX as an astrophysical process has a long history and is not restricted to X-rays. In the planetary nebula NGC 7027, the ionization structure observed with optical spectra can be explained by including CX excitation with hydrogen atoms (Pequignot et al. 1978). Chevalier et al. (1980) reported CX excited $\mathrm{H} \alpha, \mathrm{H} \beta$, and $\mathrm{H} \gamma$ lines in the supernova remnant
Tycho, which are substantially broadened by the motion of hot protons. In the UV band, Krasnopolsky \& Mumma (2001) detected prominent $\mathrm{He}$ II, C V, and O V CX lines from the comet Hyakutake, and proved that $\mathrm{CX}$ is the dominant process for the cometary EUV emission. All these CX emissions are found to be associated with outflows/winds/shocks of ionized matter which interact with the surrounding cold neutral matter at velocities of a few hundred to a few thousand kilometers per second. As reviewed by Krasnopolsky et al. (2004) and Dennerl (2010), the UV region provides a powerful but as yet not fully exploited probe of CX. So far most of the CX studies using the UV data are restricted to the nearby comets and planets.

One difficulty in CX studies in the UV band is the lack of a plasma code. A new atomic model and emission code for CX has recently been published (Gu et al. 2016a), which implements the state-of-art theoretical calculations and experimental data. It has been successfully applied to several X-ray observations (Gu et al. 2015, 2016b; Pinto et al. 2016), and to a new laboratory measurement (Shah et al. 2016). We expect that it can also stimulate new $\mathrm{CX}$ research at other wavelengths, in particular the UV band. It is hence non-trivial to verify the new code and the associated atomic data (e.g. wavelengths) before applying it to the observed UV data.

In this paper, we probe $\mathrm{CX}$ emission in the UV band from a new potential target, NGC 1275, by making full use of the new CX code. NGC 1275 is the cD galaxy of the Perseus cluster, 


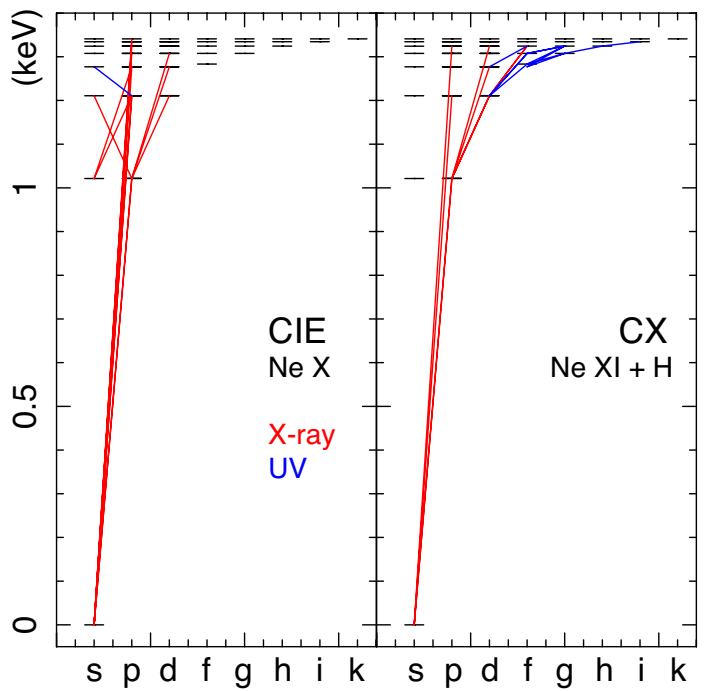

Fig. 1. Grotrian diagrams of Ne X in CIE (left) and CX (right) conditions. The same balance temperature, $k T=1 \mathrm{keV}$, is assumed in both cases. The 25 strongest transitions are marked. The red and blue lines show transitions in X-ray and UV bands, respectively. The CX model adopts the atomic-orbital close-coupling calculation in Liu et al. (2014).

which is known to host a bright and complex UV source in its core region (Baum et al. 2005). The paper is structured as follows. In Sect. 2, we present the calculation of UV lines using the new CX model. Section 3 describes the analysis and the results from the NGC 1275 UV data. The physical implications of the observed results are presented in Sect. 4 and summarized in Sect. 5. Throughout the paper, the errors are given at a $68 \%$ confidence level, and the flux is given in number of photons per second per unit area unless specifically mentioned otherwise.

\section{Charge exchange model}

Charge exchange mostly occurs at the interface of ionized and neutral particles where ions collide with atoms and capture electrons from the atoms. A unique emission line is produced when the electron, captured in an unstable level with a high principle quantum number $n$, cascades down to the ground state. The CX emission is modelled in a newly developed plasma code as introduced in Gu et al. (2015). Our code calculates the CX line flux as

$$
F=\frac{1}{4 \pi D_{1}^{2}} \int n_{\mathrm{I}} n_{\mathrm{N}} v \sigma_{\mathrm{I}, \mathrm{N}}(v, n, l, S) \mathrm{d} V,
$$

where $D_{1}$ is the luminosity distance of the object; $n_{\mathrm{I}}$ and $n_{\mathrm{N}}$ are the densities of ionized and neutral media, respectively; $v$ is the collisional velocity; $V$ is the interaction volume; and $\sigma_{\mathrm{I}, \mathrm{N}}$ is the charge exchange cross section, which highly depends on the velocity $v$ and the energy of the capture state characterized by the quantum numbers $n, l$, and $S$.

Compared to the line emission excited by free electrons, charge exchange emission shows two main features. First, $\mathrm{CX}$ shows characteristic line ratios at X-ray wavelengths. For instance, the transitions from high- $n$ excited levels to the ground are enhanced, and the forbidden-to-resonance line ratios are much higher than under the collisional ionization equilibrium (CIE) condition. This feature has been described in detail in Gu et al. (2015, 2016a,b). Second, the CX process would produce much more and brighter lines in the UV band than a CIE
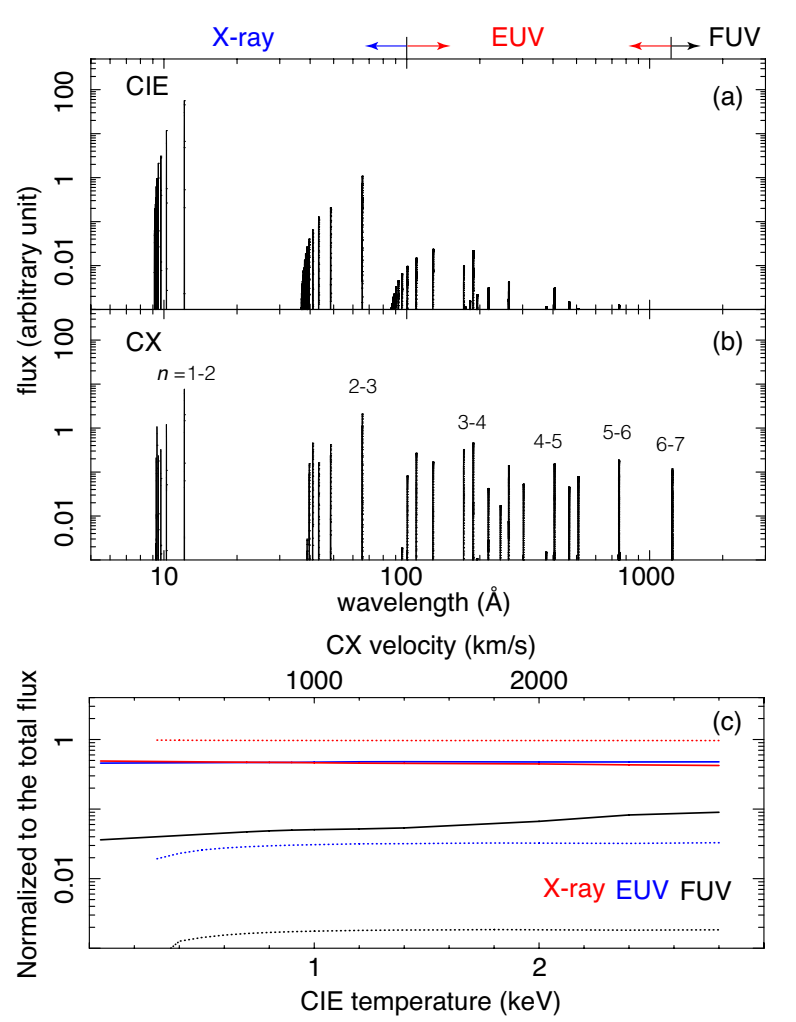

Fig. 2. a) CIE and b) CX line spectra of NeX. The CIE and CX temperature is set to $1 \mathrm{keV}$, and the CX velocity is set to $100 \mathrm{~km} \mathrm{~s}^{-1}$. The CX model is based on the AOCC calculation. c) FUV (black), EUV (blue), and X-ray (red) parts of the Ne X emission lines normalized to the total flux, plotted as a function of temperature and velocity for CIE and CX conditions, respectively. The solid and dotted lines show the $\mathrm{CX}$ and the CIE models, respectively.

would, since it introduces many transitions between two highly excited levels, while a CIE spectrum contains mostly transitions between inner-shell levels with smaller quantum number $n$ and $l$. As shown in Fig. 1, the SPEX CX model predicts that 15 of the 25 strongest Ne X CX lines are in the UV band; many of them come from large $n$ and $l$, e.g. $6 \mathrm{~h}-7 \mathrm{i}, 5 \mathrm{~g}-6 \mathrm{~h}$, and $4 \mathrm{f}-5 \mathrm{~g}$. In contrast, the strong Ne X CIE lines are mostly (24/25) in X-rays, and the emission is dominated by the $\operatorname{Ly} \alpha(1 \mathrm{~s}-2 \mathrm{p})$ transition. A direct comparison between CIE and CX spectra can be found in Fig. 2.

We further compare the total $\mathrm{CX}$ and CIE fluxes of Ne $\mathrm{X}$ in the far-UV (FUV; 1210-2000 А), extreme-UV (EUV; $100-1210 \AA$ ), and X-ray bands (1-100 $)$ ) as functions of the ion impacting velocity for $\mathrm{CX}$ and electron temperature for CIE. Based on an atomic-orbital close-coupling (AOCC; Liu et al. 2014) modelling as shown in Fig. 2, the total CX fluxes in the EUV and X-ray bands are comparable, and the FUV emission is about 5 to 15 times lower. Another physical calculation, the multichannel Landau-Zener (MCLZ; Mullen et al. 2016) method, predicts comparable X-ray and EUV fluxes to the AOCC ones. The FUV emission based on the MCLZ calculation is a factor of two higher for low-speed collisions, while a factor of eight lower at the high-velocity end than the AOCC value. The uncertainty created by the CX physical modellings is discussed in detail in Sect. 3.3. Compared to the CX case, the CIE line spectrum is dominated by X-ray emission, while the EUV and FUV contributions are only about $2-3 \%$ and $0.1-0.2 \%$ of the total flux, respectively. The UV-to-X-ray line ratios in both the 

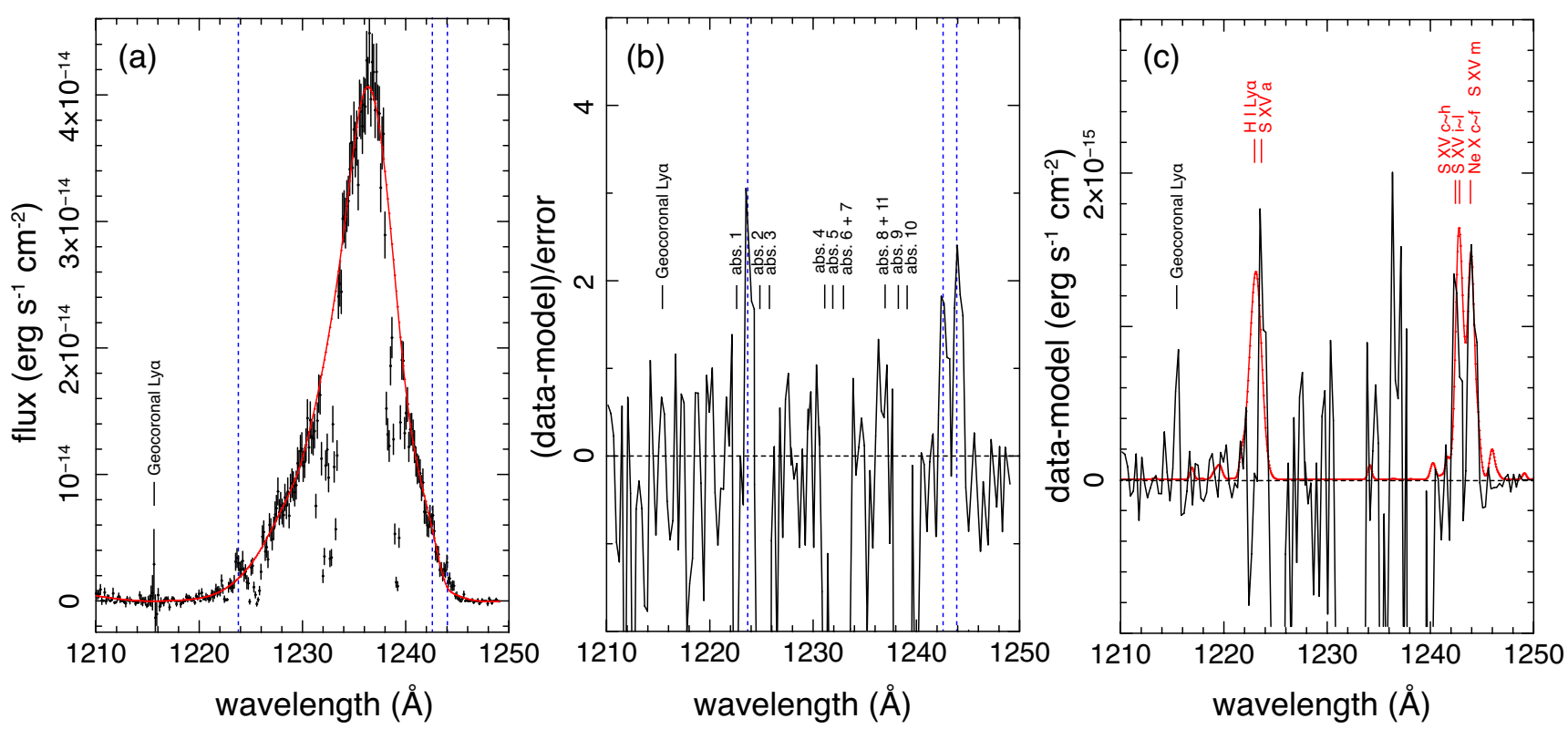

Fig. 3. a) Observed Hubble/STIS spectrum of NGC 1275 uncorrected for reddening. The red curve shows the spline fitting to the broadened Ly $\alpha$ line profile. The known features, including the geocoronal line and absorption systems reported in Baum et al. (2005) are marked in the text. The three unidentified line features are also marked with blue vertical lines. b) Significance of the emission and absorption features with respect to the spline model. c) Residual spectrum, plotted together with the CX lines in arbitrary units. The transitions of the CX lines can be found in Table 1.

$\mathrm{CX}$ and CIE conditions are relatively insensitive to the variations of velocity or temperature.

The high UV flux of the CX lines makes the UV band a unique and important window that can be used to probe CX emission from astrophysical objects. To further explore the potential of the new CX model, we apply it to an archival UV spectrum as follows.

\section{Data reduction and analysis}

The Hubble/STIS observed the NGC 1275 core on Dec. 5, 2000. The FUV Multianode Microchannel Array (MAMA) and the G140M grating were used to cover the redshifted Ly $\alpha$ line. The spectral resolution is $0.08 \AA$ FWHM, or $\sim 20 \mathrm{~km} \mathrm{~s}^{-1}$ at the cluster redshift. The observation was carried out in TIME-TAG mode to reject high sky background, and the position angle of the slit was fine-tuned using the quick-look FUV image to maximize the Ly $\alpha$ brightness. The STIS data are reduced with the standard pipeline using the updated reference files. The same spectrum was reported in Baum et al. (2005).

As shown in Fig. 3, the STIS spectrum shows a bright broadened Ly $\alpha$ line at about $1237 \AA$, with many absorption features in the wings. The ten strongest absorption features were reported in Baum et al. (2005). These features have H I column densities of $10^{12}-10^{14} \mathrm{~cm}^{-2}$, velocity shifts of $-3543 \mathrm{~km} \mathrm{~s}^{-1}$ to $533 \mathrm{~km} \mathrm{~s}^{-1}$ with respect to the nucleus, and line widths of $15-80 \mathrm{~km} \mathrm{~s}^{-1}$. Baum et al. (2005) suggested that the column densities, shifts, and widths are consistent with the intrinsic absorption features produced by clouds in the nucleus outflow.

To reveal the additional emission features, it is essential to remove the primary $\operatorname{Ly} \alpha$ line profile and any other continuum features. Here we fit these features with a phenomenological ten-knot spline function, and subtract it from the spectrum. The spline-fitting process has been applied in many previous AGN studies using the UV spectra (Kraemer et al. 2001a,b; Shull et al. 2012; Stevans et al. 2014). As shown in these works, the spline function can provide a reasonable fit to the smoothly varying continuum, and to the strongly broadened line features. In the fitting, the bands containing the ten strong absorption features are discarded. The ten spline knots are spread evenly across the absorption-free bands, and are adjusted manually to optimize the fitting statistics. The best-fit $\chi^{2}$ is 270 for 231 degrees of freedom. As shown in Fig. 3, the observed Ly $\alpha$ line profile is in general well described by the spline function, except for a few excesses at around $1224 \AA, 1237 \AA$, $1242 \AA$, and $1244 \AA$. The positive residual at $1237 \AA$ indicates a more cuspy Ly $\alpha$ profile than the best-fit model. We do not investigate this substructure further as it is not the aim of this paper. To exclude all possible line features, we discarded the four bands that contains excesses, and rerun the spline fit. This gives a best-fit $\chi^{2}$ of 222 for 206 degrees of freedom. The best-fit spline is used as a baseline model for the line detection.

By varying the spline parameters within their error ranges, we find that the model has a $4 \%$ uncertainty at $1210 \AA, 3 \%$ at $1224 \AA, 2 \%$ at $1237 \AA$, and $3 \%$ at $1244 \AA$. To further assess the uncertainty due to the model setting, we test to fit the data with a 15-knot spline function. The best-fit statistics, $\chi^{2}$ of 213 for 201 degrees of freedom, is roughly consistent with that of the baseline model. The new spline deviates by $2-4 \%$ from the baseline. Hence, we consider a systematic uncertainty of $4 \%$, which is combined with the statistical uncertainty to derive the total error of the data.

As plotted in Fig. 3, after subtracting the baseline model, the residual spectrum unveils the line-like features at around $1224 \AA, 1242 \AA$, and $1244 \AA$, with peak significance of $3.0 \sigma$, $1.8 \sigma$, and $2.3 \sigma$, respectively. The other excesses, including that at $1237 \AA$, are all around or below $1 \sigma$. To verify the possible features, we fit the absorption-free band with the baseline spline and three additional Gaussian functions. The new fit gives a $\chi^{2}$ of 240 for degrees of freedom of 222, which is a significant improvement on the original fit (270/231). An F-test shows that it is unlikely (null hypothesis probability $=2 \times 10^{-3}$ ) that the improvement is achieved by chance. The best-fit central energies of the Gaussian components are $1223.6 \pm 0.4 \AA$, 

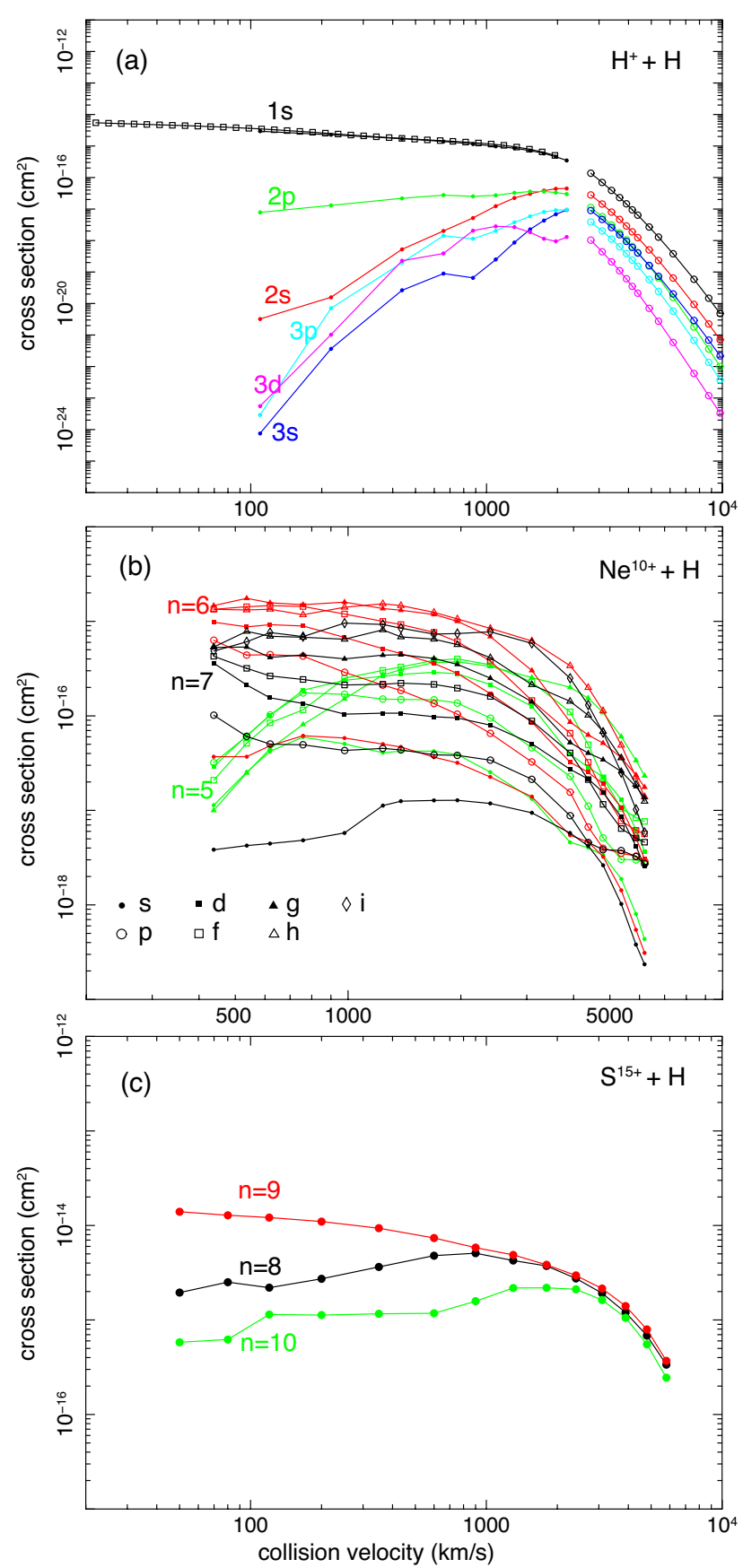

Fig. 4. a) $n l$-resolved $\mathrm{CX}$ cross sections of the proton-H atom collision. The data shown by dots, circles, and squares are taken from the calculations by Harel et al. (1998), Belkić et al. (1992), and Belkić (1991), respectively. b), c) Cross sections of bare $\mathrm{Ne}$ and $\mathrm{H}$-like $\mathrm{S}$ colliding with atomic hydrogen. The Ne and $\mathrm{S}$ data are derived via the AOCC method (Cumbee et al. 2016) and extrapolation based on data compilation (Gu et al. 2016a), respectively.

$1242.4 \pm 0.5 \AA$, and $1244.0 \pm 0.2 \AA$, which give fluxes of $(1.0 \pm 0.4) \times 10^{-15} \mathrm{erg} \mathrm{s}^{-1} \mathrm{~cm}^{-2},(0.6 \pm 0.3) \times 10^{-15} \mathrm{erg} \mathrm{s}^{-1} \mathrm{~cm}^{-2}$, and $(0.8 \pm 0.3) \times 10^{-15} \mathrm{erg} \mathrm{s}^{-1} \mathrm{~cm}^{-2}$, respectively. The total flux is about $2 \%$ of the primary Ly $\alpha$ feature.

So far the detection is essentially based on a blind line search, in which the probability of a false detection caused by positive random fluctuation must be taken into account (the "lookelsewhere" effect; Gross \& Vitells 2010). Considering that the total bin width of the three weak features are about $1 / 20$ of the absorption-free band, the look-elsewhere effect would boost the null hypothesis probability from $2 \times 10^{-3}$ to $4 \times 10^{-2}$. If these features are indeed identified as atomic lines, we then know the exact line energies, and the look-elsewhere effect can be reduced. Hence, we compare these features with the atomic line list as follows.

\subsection{Line identification}

First we search for the target lines in the line list of CIE emission. Since the emission might come from any object, including the cool clouds, the ISM, and the ICM in the central region of NGC 1275, we look into lines in the temperature range of 0.05 to $5 \mathrm{keV}$. Assuming that the clouds might have a line-ofsight velocity of $-3543 \mathrm{~km} \mathrm{~s}^{-1}$ to $533 \mathrm{~km} \mathrm{~s}^{-1}$ with respect to the nucleus (Baum et al. 2005), we consider a redshift range of 0.00573 to 0.01933 , which corresponds to a rest-frame wavelength of 1200-1237 $\AA$. The flux threshold is set to be $1 \%$ of the $\mathrm{HI}_{\mathrm{I}} \mathrm{Ly} \alpha$ line at rest-frame $1215.7 \AA$. In this band, only a few emission lines are present; they are listed in Table 1 at three representative temperatures. At a low temperature of $0.05 \mathrm{keV}$, He II, and OV lines appear from $1213 \AA$ to $1218 \AA$, while at $1 \mathrm{keV}$ the Cr XX line can be found at $1205 \AA$. Most of these lines, except the He II line at around $1215 \AA$, disappear at a higher plasma temperature. It is therefore unlikely that the observed features at $1223.6 \AA, 1242.4 \AA$, and $1244.0 \AA$ can be explained simultaneously by one thermal emission component, even if the possible line-of-sight velocity is taken into account, nor can these features be ascribed to CIE emission of local Galactic or geocoronal sources.

Next we explore the photoionization model. Here we compute a spectrum assuming photoionization equilibrium based on the new pion model in SPEX v3.03. The ionizing radiation from the AGN continuum components is approximated by a powerlaw model with a photon index of 2, spanning from $0.1 \mathrm{eV}$ to $1 \mathrm{MeV}$. As shown in Mehdipour et al. (2016), such an ionizing model can roughly reproduce the ionization balance of the photoionized plasma in typical Type 1 AGNs. Then we calculate the spectrum by changing the ionization parameter $\xi$ gradually from $10^{-2}$ to $10^{4}$, corresponding to a balance temperature from about $10^{-3} \mathrm{keV}$ to $10 \mathrm{keV}$. The resulting lines in the band of interest are identical to those of the CIE plasma, as listed in Table 1. This means that the observed weak features cannot be fully ascribed to a photoionization component.

Finally, we investigate the charge exchange model. Similar to the thermal case, we scan the CX line list by changing the ionization state of the projectile ions from 0.05 to $5 \mathrm{keV}$. Since the CX emission also varies as a function of collision velocity, we explore a velocity range of $50 \mathrm{~km} \mathrm{~s}^{-1}$ to $2000 \mathrm{~km} \mathrm{~s}^{-1}$ to include all the possible transitions. As shown in Table 1, the CX model predicts rich emission lines at an ionization temperature of $\sim 1 \mathrm{keV}$. The related lines are the cascade emissions of $\mathrm{HI}, \mathrm{NeX}$, and SXV ions. The H I Ly $\alpha$ transition is created by proton-atom collisions. As seen in Fig. 4, most of the recombined electrons are captured directly into the ground state as the projectile and target atomic nuclei have essentially the same potential. Then the Ly $\alpha$ line becomes significant only when the collision dynamics is important, i.e. velocity $\geq 1000 \mathrm{~km} \mathrm{~s}^{-1}$. The same condition is applied to He II $n=4$ to $n=2$ transitions at $\sim 1215.1 \AA$ (Table 1), which is not yet included in the current CX model in SPEX due to the lack of theoretical and experimental data of the bare $\mathrm{He}+$ atomic $\mathrm{H}$ collision. It is expected that some dynamical input is required to capture an electron onto $n=4$ of He II, which has a larger potential than the ground state 
Table 1. Related emission lines.

\begin{tabular}{|c|c|c|c|c|c|c|}
\hline Type & $k T(\mathrm{keV})^{a}$ & Name & Lower level & Upper level & Wavelength $(\AA)$ & Fluxes $^{b}$ \\
\hline CIE & 0.05 & He II & $2 \mathrm{~s}^{2} \mathrm{~S}_{1 / 2}$ & $4 p^{2} \mathrm{P}_{3 / 2}$ & 1215.095 & 0.013 \\
\hline CIE & 0.05 & He II & $2 p^{2} \mathrm{P}_{3 / 2}$ & $4 d^{2} D_{5 / 2}$ & 1215.171 & 0.010 \\
\hline CIE & 0.05 & $\mathrm{OV}$ & $2 \mathrm{~s}^{2}{ }^{1} \mathrm{~S}_{0}$ & $2 \mathrm{~s} .2 \mathrm{p}{ }^{3} \mathrm{P}_{2}$ & 1213.799 & 0.047 \\
\hline CIE & 0.05 & $\mathrm{OV}$ & $2 \mathrm{~s}^{2}{ }^{1} \mathrm{~S}_{0}$ & $2 \mathrm{~s} .2 \mathrm{p}^{3} \mathrm{P}_{1}$ & 1218.335 & 0.030 \\
\hline$\overline{\mathrm{CIE}}$ & 1.0 & He II & $2 \mathrm{~s}^{2} \mathrm{~S}_{1 / 2}$ & $4 p^{2} P_{3 / 2}$ & 1215.095 & 0.010 \\
\hline CIE & 1.0 & $\mathrm{CrXX}$ & $2 \mathrm{~s}^{2} .2 \mathrm{p}^{2} \mathrm{P}_{1 / 2}$ & $2 \mathrm{~s}^{2} .2 \mathrm{p}^{2} \mathrm{P}_{3 / 2}$ & 1205.252 & 0.033 \\
\hline $\mathrm{CIE}$ & 5.0 & He II & $2 \mathrm{~s}^{2} \mathrm{~S}_{1 / 2}$ & $4 p^{2} \mathrm{P}_{3 / 2}$ & 1215.095 & 0.010 \\
\hline $\mathrm{CX}$ & 0.05 & $\mathrm{OV}$ & $2 \mathrm{~s}^{2}{ }^{1} \mathrm{~S}_{0}$ & $2 \mathrm{~s} .2 \mathrm{p}^{3} \mathrm{P}_{2}$ & 1213.799 & 0.329 \\
\hline $\mathrm{CX}$ & 0.05 & $\mathrm{Ov}$ & $2 \mathrm{~s}^{2}{ }^{1} \mathrm{~S}_{0}$ & $2 \mathrm{~s} .2 \mathrm{p}^{3} \mathrm{P}_{1}$ & 1218.335 & 0.207 \\
\hline $\mathrm{CX}$ & 1.0 & $\mathrm{NeX} \mathrm{a}$ & $6 f^{2} F_{5 / 2}$ & $7 \mathrm{~g}^{2} \mathrm{G}_{7 / 2}$ & 1236.063 & 0.084 \\
\hline CX & 1.0 & $\mathrm{Ne} \times \mathrm{b}$ & $6 d^{2} D_{5 / 2}$ & $7 \mathrm{f}^{2} \mathrm{~F}_{7 / 2}$ & 1236.064 & 0.031 \\
\hline CX & 1.0 & $\mathrm{NeX} \mathrm{c}$ & $6 \mathrm{~g}^{2} \mathrm{G}_{7 / 2}$ & $7 h^{2} \mathrm{H}_{9 / 2}$ & 1236.278 & 0.109 \\
\hline CX & 1.0 & $\mathrm{NeX} \mathrm{d}$ & $6 \mathrm{f}^{2} \mathrm{~F}_{7 / 2}$ & $7 \mathrm{~g}^{2} \mathrm{G}_{9 / 2}$ & 1236.278 & 0.269 \\
\hline CX & 1.0 & $\mathrm{NeX} \mathrm{e}$ & $6 \mathrm{~h}^{2} \mathrm{H}_{9 / 2}$ & $7 \mathrm{i}^{2} \mathrm{I}_{11 / 2}$ & 1236.398 & 0.541 \\
\hline CX & 1.0 & $\mathrm{Ne} X \mathrm{f}$ & $6 \mathrm{~g}^{2} \mathrm{G}_{9 / 2}$ & $7 h^{2} \mathrm{H}_{11 / 2}$ & 1236.398 & 0.330 \\
\hline CX & 1.0 & $\mathrm{SXV}$ a & $1 \mathrm{~s} .8 \mathrm{p}{ }^{3} \mathrm{P}_{2}$ & $1 \mathrm{~s} .9 \mathrm{~d}^{3} \mathrm{D}_{3}$ & 1216.128 & 0.133 \\
\hline CX & 1.0 & $S x V b$ & 1s. $8 \mathrm{p}^{1} \mathrm{P}_{1}$ & $1 \mathrm{~s} .9 \mathrm{~d}^{1} \mathrm{D}_{2}$ & 1232.703 & 0.094 \\
\hline CX & 1.0 & $S x V c$ & $1 \mathrm{~s} .8 \mathrm{~g}^{3} \mathrm{G}_{3}$ & 1s. $9 \mathrm{~h}^{3} \mathrm{H}_{4}$ & 1234.939 & 0.124 \\
\hline CX & 1.0 & $\mathrm{SXV} \mathrm{d}$ & $1 \mathrm{~s} .8 \mathrm{~g}^{3} \mathrm{G}_{4}$ & 1s. $9 \mathrm{~h}^{3} \mathrm{H}_{5}$ & 1234.940 & 0.156 \\
\hline CX & 1.0 & SXV e & $1 \mathrm{~s} .8 \mathrm{f}^{3} \mathrm{~F}_{2}$ & $1 \mathrm{~s} .9 \mathrm{~g}{ }^{3} \mathrm{G}_{3}$ & 1235.132 & 0.161 \\
\hline CX & 1.0 & $S X V f$ & $1 \mathrm{~s} .8 \mathrm{f}^{3} \mathrm{~F}_{3}$ & $1 \mathrm{~s} .9 \mathrm{~g}^{3} \mathrm{G}_{4}$ & 1235.152 & 0.217 \\
\hline $\mathrm{CX}$ & 1.0 & $\mathrm{SxV} g$ & $1 \mathrm{~s} .8 \mathrm{~g}^{3} \mathrm{G}_{5}$ & $1 \mathrm{~s} .9 \mathrm{~h}^{3} \mathrm{H}_{6}$ & 1235.175 & 0.188 \\
\hline CX & 1.0 & S XVh & $1 \mathrm{~s} .8 \mathrm{~g}^{1} \mathrm{G}_{4}$ & $1 \mathrm{~s} .9 \mathrm{~h}{ }^{1} \mathrm{H}_{5}$ & 1235.175 & 0.156 \\
\hline CX & 1.0 & $\mathrm{SxV}$ i & $1 \mathrm{~s} .8 \mathrm{~h}^{3} \mathrm{H}_{6}$ & $1 \mathrm{~s} .9 \mathrm{i}^{3} \mathrm{I}_{7}$ & 1235.341 & 0.081 \\
\hline CX & 1.0 & $\mathrm{~S} \times \mathrm{XV}$ & $1 \mathrm{~s} .8 \mathrm{f}{ }^{3} \mathrm{~F}_{4}$ & $1 \mathrm{~s} .9 \mathrm{~g}^{3} \mathrm{G}_{5}$ & 1235.552 & 0.276 \\
\hline $\mathrm{CX}$ & 1.0 & S XV k & $1 \mathrm{~s} .8 \mathrm{f}^{1} \mathrm{~F}_{3}$ & $1 \mathrm{~s} .9 \mathrm{~g}^{1} \mathrm{G}_{4}$ & 1235.579 & 0.219 \\
\hline CX & 1.0 & S XV 1 & $1 \mathrm{~s} .8 \mathrm{~d}^{3} \mathrm{D}_{1}$ & $1 \mathrm{~s} .9 \mathrm{f}^{3} \mathrm{~F}_{2}$ & 1236.102 & 0.119 \\
\hline CX & 1.0 & $\mathrm{SXV} \mathrm{m}$ & $1 \mathrm{~s} .8 \mathrm{~d}^{3} \mathrm{D}_{2}$ & $1 \mathrm{~s} .9 \mathrm{f}^{3} \mathrm{~F}_{3}$ & 1236.440 & 0.163 \\
\hline $\mathrm{CX}$ & 5.0 & $\mathrm{NeX}$ & $6 f^{2} F_{7 / 2}$ & $7 \mathrm{~g}^{2} \mathrm{G}_{9 / 2}$ & 1236.278 & 0.020 \\
\hline CX & 5.0 & $\mathrm{NeX}$ & $6 \mathrm{~h}^{2} \mathrm{H}_{9 / 2}$ & $7 \mathrm{i}^{2} \mathrm{I}_{11 / 2}$ & 1236.398 & 0.025 \\
\hline CX & 5.0 & $\mathrm{Ne} X$ & $6 \mathrm{~g}^{2} \mathrm{G}_{9 / 2}$ & $7 \mathrm{~h}^{2} \mathrm{H}_{11 / 2}$ & 1236.398 & 0.040 \\
\hline
\end{tabular}

Notes. ${ }^{(a)}$ The balance temperature used in the calculation. The CX collision velocity is set to $500 \mathrm{~km} \mathrm{~s}^{-1}$. ${ }^{(b)}$ Line fluxes normalized to that of the H I Ly $\alpha$.

of a donor hydrogen atom. Hence we treat the He II $1215.1 \AA$ line as an additional flux of the $\mathrm{H} \mathrm{I}$ Ly $\alpha$ line, due to their similar properties and proximity in wavelength.

The NeX $n=7$ to $n=6$ transitions at $\sim 1236 \AA$, and S XV $n=9$ to $n=8$ complex at 1216-1236 $\AA$ are all characteristic $\mathrm{CX}$ lines which are excited by bare $\mathrm{Ne}$ and $\mathrm{H}$-like $\mathrm{S}$ collisions with neutral atoms, respectively. In Fig. 4 we demonstrate that the upper levels of the related transitions can be populated effectively by the CX. For interactions between bare $\mathrm{Ne}$ and atomic hydrogen, most of the captured electrons are expected to fall onto $n=6$ and $n=7$; the two levels take about $70 \%$ and $30 \%$ of the total capture at $v=100 \mathrm{~km} \mathrm{~s}^{-1}$, and about $60 \%$ and $30 \%$ at $v=1000 \mathrm{~km} \mathrm{~s}^{-1}$. For $\mathrm{H}$-like sulfur $+\mathrm{H}$ charge exchange, the $n=9$ is the dominant level, contributing to about $80 \%$ of the total capture at a few hundred $\mathrm{km} \mathrm{s}^{-1}$, and at $v=1000 \mathrm{~km} \mathrm{~s}^{-1}$, the $n=8$ and $n=9$ have a similar share, about $40 \%$ of the total capture (Fig. 4). The two projectile ions, bare $\mathrm{Ne}$ and $\mathrm{H}$-like $S$, require ionization temperatures of $\geq 0.2 \mathrm{keV}$ and $\geq 0.6 \mathrm{keV}$, respectively (see also Fig. 6).

The STIS spectrum does not show the CX features at the systemic velocity of NGC 1275. By shifting the CX lines within the velocity range of the observed Ly $\alpha$ absorbers, i.e. $-3543 \mathrm{~km} \mathrm{~s}^{-1}$ to $533 \mathrm{~km} \mathrm{~s}^{-1}$ with respect to the galaxy core, we find one potential match at a relative velocity of about $-3400 \mathrm{~km} \mathrm{~s}^{-1}$, as the H I Ly $\alpha$, He II $n=4$ to $n=2$, and S XV 1s.8p $\left({ }^{3} \mathrm{P}_{2}\right)-1 \mathrm{~s} .9 \mathrm{~d}\left({ }^{3} \mathrm{D}_{3}\right)$ transitions would then appear at 1222.7-1223.7 $\AA$, a complex of S XV $n=9$ to $n=8$ transitions at $\sim 1242.4 \AA$ (S XV c-h in Table 1), and the $\operatorname{NeX} n=7$ to $n=6$ lines and one S XV line at around $1244.0 \AA$ (Ne X c-f and S XV m in Table 1). As shown in Fig. 3, the three putative features can be simultaneously explained by the $\mathrm{CX}$ lines emitted at a high line-of-sight velocity relative to the nucleus.

A remaining issue with this picture is that the peak of the model for the possible feature at $1223.6 \AA$ is slightly offset to shorter wavelengths with respect to the data (Fig. 3). This might be explained by the fact that the expected relative velocity of the $\mathrm{CX}$ emitter is quite close to one of the Ly $\alpha$ absorbers discovered in Baum et al. (2005; No.1 in their Table 4), so that the blue wing of the 1222.7-1223.7 $\AA$ CX complex might have been partially absorbed. We will present more insights on the relation between CX emitter and Ly $\alpha$ absorbers in Sect. 4.1.

Since the CX line energies are not determined by the natural redshift, but instead by a scan across the radial velocity range, the 


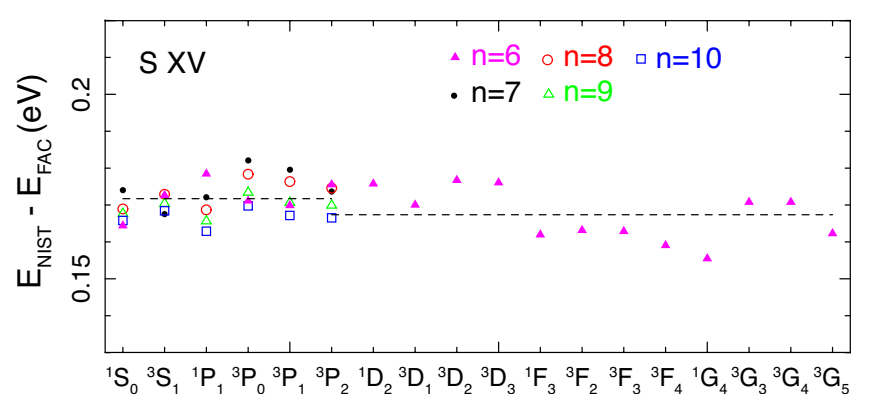

Fig. 5. Differences between level energies from NIST database and FAC calculations for $n=6-10$ of S XV. The two dashed lines show the average offsets for $l=0$ and 1 , and for $l=2-4$.

look-elsewhere effect cannot be totally eliminated. Taking into account the bin width of the observed weak line features and the assumed velocity range, it would take about seven independent steps to move the CX line template through the absorption-free band of the observed data; therefore, the null hypothesis probability obtained in Sect. 3 should be increased to $1.4 \times 10^{-2}$ by the look-elsewhere effect.

When shifting the CX model to a line-of-sight velocity of $6595 \mathrm{~km} \mathrm{~s}^{-1}$, i.e. $1334 \mathrm{~km} \mathrm{~s}^{-1}$ higher than that of NGC 1275, we can obtain an alternative potential match of two features. The H I Ly $\alpha$ and S XV $1 \mathrm{~s} .8 \mathrm{p}\left({ }^{3} \mathrm{P}_{2}\right)-1 \mathrm{~s} .9 \mathrm{~d}\left({ }^{3} \mathrm{D}_{3}\right)$ lines would be seen at 1242.4-1242.9 $\AA$, and a Fe XXIV $n=12$ to $n=11 \mathrm{CX}$ complex would appear at $\sim 1223.9 \AA$. Since the third feature at $1244.0 \AA$ cannot be explained in this setting, it becomes less favourable than the previous one.

The above CX model assumes that each ion experiences one charge exchange along the line of sight. In the case of a very dense cloud, an impinging highly ionized particle might undergo multiple CX with the cloud neutrals until it reaches a neutral state. In that case, many transitions of lowly ionized ions would appear in the spectrum. We find that two strong $\mathrm{O} V$ lines, $2 \mathrm{~s}^{2}\left({ }^{1} \mathrm{~S}_{0}\right)-2 \mathrm{~s} .2 \mathrm{p}\left({ }^{3} \mathrm{P}_{2}\right)$ and $2 \mathrm{~s}^{2}\left({ }^{1} \mathrm{~S}_{0}\right)-2 \mathrm{~s} .2 \mathrm{p}\left({ }^{3} \mathrm{P}_{1}\right)$ at $1213.8 \AA$ and $1218.3 \AA$, would dominate the model spectrum in the UV band. The two lines do not match with any observed features, and hence the present single collision is much more likely than the multiple collision model.

\subsection{Wavelength uncertainties}

Since the related lines include transitions between two highly excited levels, it is crucial to examine the uncertainties of the transition energies. The H I data are taken from the calculated values of Erickson (1977). By comparing these data with the data compiled in Kramida (2010), we find that the uncertainties are less than $0.00001 \mathrm{eV}$, and the induced error on the Ly $\alpha$ transition is $\sim 0.001 \AA$. The energy level data from Erickson (1977) are also used for the Ne X lines. Here we have cross-checked them with the critically revised data from the latest NIST database (Kramida et al. 2015), and found a $0.0615 \pm 0.0001 \mathrm{eV}$ energy shift between the two sets. Since this shift is nearly constant for all levels, it will not affect the accuracy of the transitions between two excited levels. Hence the induced wavelength uncertainties are quite small, $\sim 0.01 \AA$, for the Ne X CX lines.

For S XV, we use the measured values of Martin et al. (1990; available only for quantum number $l=0$ and 1 in $n \geq 7$ ) which are present in the latest NIST database, and derive for the rest levels using the Flexible Atomic Code (FAC; Gu 2008). The measured values have uncertainties of $0.001 \mathrm{eV}$. To minimize the
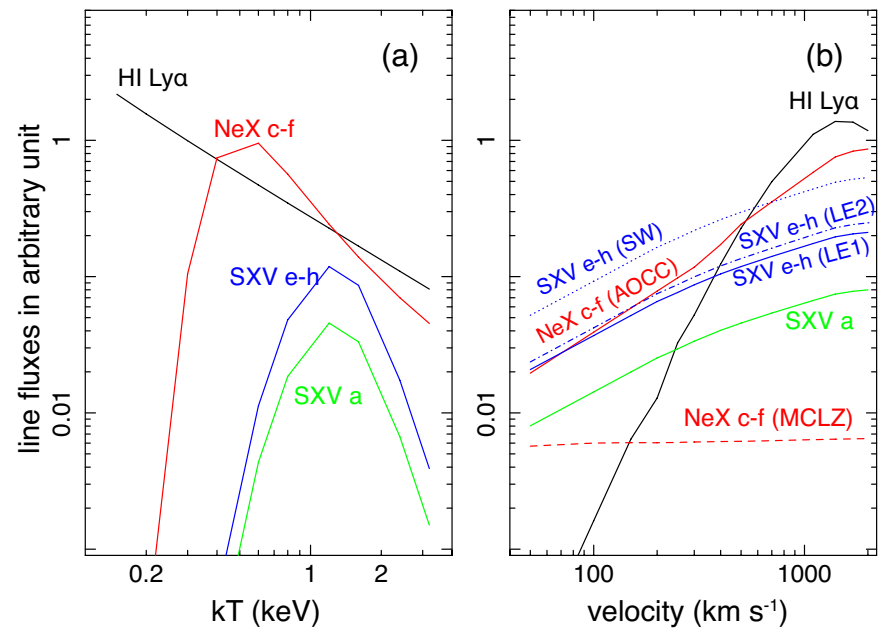

Fig. 6. a) $\mathrm{CX}$ line fluxes as a function of ionization temperature of the impinging ions. The detailed transitions can be found in Table 1. The collision velocity is set to be $500 \mathrm{~km} \mathrm{~s}^{-1}$. b) CX line fluxes as a function of collision velocities. The ionization temperature of the projectile ions are set to be $1.2 \mathrm{keV}$. The solid and dashed red lines show two different calculations of the Ne X CX. The solid, dotted, and dash-dotted blue lines are the S XV CX lines assuming $l$-population functions of low-energy I (LE1), statistical weight (SW), and low-energy II (LE2), respectively (see Gu et al. 2016a, for their forms).

deviation between the two data sources, we further calibrate the FAC values to the experimental results. As shown in Fig. 5, the raw FAC calculations for $l=0$ and 1 are systematically smaller than the experimental values by $0.174 \pm 0.001 \mathrm{eV}$ at $n=8$, and $0.167 \pm 0.001 \mathrm{eV}$ at $n=9$. For reference, the systematic deviations are $0.172 \pm 0.002 \mathrm{eV}$ at $n=7$, and $0.167 \pm 0.001 \mathrm{eV}$ at $n=10$. We also compare the FAC energies of $l=2-4$ of $n=6$ with the measured values, and find a systematic offset of $0.167 \pm 0.003 \mathrm{eV}$. Assuming that the FAC values for $l>1$ levels of $n=8$ and 9 share the same average offset as $n=6$, we apply a $n l$-dependent correction to the FAC calculations. The corrected energies agree well with the experimental data within a scatter of about $0.002 \mathrm{eV}$ and $0.003 \mathrm{eV}$ for $l \leq 1$ and $l>1$, respectively. Hence the final wavelength uncertainties of the $\operatorname{SXV} n=9$ to $n=8$ lines are about $0.2 \AA$ for $l=0$ and 1 , and $0.3 \AA$ for levels with larger $l$.

\subsection{Intensity uncertainties}

In the current model, the relative intensities of the CX lines are rather uncertain, since these lines are highly sensitive to (1) ionization states and abundances of the projectile ions; (2) the collision velocity; (3) the theoretical method for CX calculation and the $l$-distribution function of the captured electron; and (4) the type of target atoms. None of these properties is known for this object. In Fig. 6, we show the intensities of four representative lines/complexes, i.e. H I Ly $\alpha$ at $1215.67 \AA$, the $\mathrm{NeX} n=7$ to $n=6$ complex at $\sim 1236.3 \AA$, S XV 1s.8p $\left({ }^{3} \mathrm{P}_{2}\right)-1 \mathrm{~s} .9 \mathrm{~d}\left({ }^{3} \mathrm{D}_{3}\right)$ at $1216.13 \AA$, and the $\mathrm{S} \mathrm{XV} n=9$ to $n=8$ complex at $\sim 1235.1 \AA$, as a function of the ionization temperature. The newly updated ionization balance of Urdampilleta (2017) and the proto-solar abundance of Lodders \& Palme (2009) are utilized in the calculation. For a temperature range from 0.5 to $1.0 \mathrm{keV}$, the intensities of $\mathrm{NeX}$ and $\mathrm{SXV}$ lines change by a factor of 5 and 250 , respectively. For item (2), we calculate the four sets of lines with the $\mathrm{CX}$ velocity changing from $50 \mathrm{~km} \mathrm{~s}^{-1}$ to $2000 \mathrm{~km} \mathrm{~s}^{-1}$. As 
shown in Fig. 6, the HI, Ne X, and two S XV features vary dramatically by respective factors of about 9000,50 , and 10 over the velocity range.

The CX model also highly depends on the theoretical method utilized in the rate calculation. As shown in Cumbee et al. (2016) and Mullen et al. (2016), the fluxes of high- $n$ transitions differ in general by factors of $2-5$ in different $\mathrm{CX}$ calculations. For Ne X $n=7$ to $n=6$ transitions at $\sim 1236.3 \AA$, the calculations by the AOCC method differs by a factor of 3 to 130 from the values by the MCLZ method (Fig. 6). The large discrepancy is due to the fact that the MCLZ calculation prefers a peak of electron capture at $n=6$, while the AOCC gives a flatter distribution over $n=5,6$, and 7 . On the other hand, the assumed $l$-distribution of the captured electron will also strongly affect the line fluxes. As shown in Fig. 6, the distribution function based on statistical weight gives the largest S XV line flux since it can effectively populate the large $l$ shells, which are essentially the upper levels of the $1235.1 \AA$ transitions. The line flux by the more commonly used low-energy I weighting function is lower by a factor of $\sim 2.5$. Finally, for item (4), the properties of the target atoms, in particular the ionization potential, might influence the $n$ distribution of the captured electrons (Eq. (3) in Gu et al. 2016a). Mullen et al. (2016) showed that the Fe XXVI CX lines from an atomic $\mathrm{He}$ donor can vary by a factor of $\sim 10-100$ relative to a $\mathrm{H}$ target.

As we have shown above, the factors governing the CX line intensities are manifold and highly degenerate. Given the current data, it is not possible to untangle these effects. More observations of CX transitions from the same ions at other wavelengths are needed to resolve the degeneracy.

\section{Discussion}

Based on a new CX model, we prove that the CX process can naturally produce transitions between large $n l$ levels. Many of these transitions fall in the UV range, making it a powerful band with which to detect and diagnose the astrophysical CX emission. By reanalysing the archival Hubble/STIS spectrum of the core of NGC 1275 in the Perseus cluster, we identified three weak lines at about $1223.6 \AA$, $1242.4 \AA$, and $1244.0 \AA$, each with a significance of $2-3 \sigma$. The putative features are best explained by a CX emission model, with a line-of-sight velocity offset of about $-3400 \mathrm{~km} \mathrm{~s}^{-1}$ with respect to the NGC 1275 nucleus, which resembles one of the Ly $\alpha$ absorbers reported in Baum et al. (2005). In this model, the lines are emitted when bare hydrogen, neon, and $\mathrm{H}$-like sulfur ions collide with neutral matter. The most recent atomic database gives a wavelength uncertainty of $\leq 0.2 \AA$ for these CX transitions. Owing to the model degeneracy, the observed line intensities cannot be used for further interpretation.

\subsection{Origin of the putative charge exchange component}

First we consider the possibility that the $\mathrm{CX}$ emission originates from the hot intracluster medium (ICM) interacting with the $\mathrm{H} \alpha$ emission filaments along the line of sight of NGC 1275 (Conselice et al. 2001). Walker et al. (2015) showed that some of the X-ray emission from the filaments might indeed be CX lines. However, these filaments have relative velocities of roughly -300 to $300 \mathrm{~km} \mathrm{~s}^{-1}$ with respect to the core, while our model indicates a relative velocity of $-3400 \mathrm{~km} \mathrm{~s}^{-1}$, which means that the $\mathrm{H} \alpha$ filaments cannot be the main CX source.

Interactions between the ICM and the cold gas halos of member galaxies might also produce CX emission (Gu et al. 2013, 2015). Similar interaction is also expected when field galaxies or galaxy groups are merging or infalling into the cluster. By searching the SIMBAD database for member galaxies within $5^{\prime}$ along the line of sight of NGC 1275, we find that the galaxies cover a relative velocity range of $-2400 \mathrm{~km} \mathrm{~s}^{-1}$ to $3000 \mathrm{~km} \mathrm{~s}^{-1}$, none of them can match with the putative CX component. Thus, the possible CX emission cannot be associated with the member galaxies.

Another possible CX source is the accretion of matter with low ionization state onto the cluster outskirt, which collides with the hot ions energized by the accretion shocks. However, numerical simulations show that the velocities of the accretion flow are normally $<1000 \mathrm{~km} \mathrm{~s}^{-1}$ (Vazza et al. 2011; Schaal et al. 2016), which does not match the observed velocity offset.

One remaining possibility that might apply to the charge exchange component is that it comes from interactions between the AGN outflow gas and the surrounding matter. A similar outflow might be responsible for the absorption features in the wing of the $\operatorname{Ly} \alpha$ line reported in Baum et al. (2005). In fact, outflows have been detected extensively in the UV spectra of Seyferts, many of them have similar column densities and velocity offsets to those in NGC 1275 (e.g. Kriss et al. 2000). Kriss et al. (2011) have reported that the AGN outflow wind might be in multiple ionization states, which are determined by the balance between time-variable photoionization and recombination. Some clouds might be highly ionized, and they can emit CX emission by interacting with the torus and/or molecular accretion disk, although the observed velocities of the accreting molecular gas in NGC 1275 are $\leq 1000 \mathrm{~km} \mathrm{~s}^{-1}$ (Scharwächter et al. 2013). In the case of cold outflows, the CX lines would be seen when the wind penetrates into the hot medium surrounding the nucleus. A similar condition has been recently discovered in a Seyfert I AGN NGC 5548 (Mao, in prep.): a CX N VII X-ray line is detected with the XMM-Newton RGS, and the CX emitter is likely to be one of the ionized outflowing clouds in that system.

In the outflow scenario, the collision velocity between hot and cold matter should be of the same order as the observed velocity offset $\left(\sim 3400 \mathrm{~km} \mathrm{~s}^{-1}\right)$, which is higher than the collision velocity assumed in the current CX model in Table 1 . At such a high collision velocity, the H I Ly $\alpha$ and Ne X lines at $1223.6 \AA$ and $1244.0 \AA$ are expected to become much brighter than the S XV lines at 1242.4 $\AA$ (Fig. 6). However, owing to the large uncertainties in the CX modelling (Sect. 3.3) and in the observed data (Fig. 3), it is practically not possible to derive any useful constraints on the collision velocity for this object.

If the observed emission line features are indeed related to one of the ten outflowing Ly $\alpha$ absorbers reported in Baum et al. (2005), we might expect similar phenomenon from the other absorbers. Absorbers 2 and 3 in Baum et al. (2005) have velocity offsets of about $-3000 \mathrm{~km} \mathrm{~s}^{-1}$ and $-2800 \mathrm{~km} \mathrm{~s}^{-1}$, respectively. The possible CX lines associated with these two absorbers will be at 1224.6-1225.6 $\AA$ (H I and S XV), 1244.0-1245.0 $\mathrm{A}$ (S XV), and 1245.8-1246.6 $\AA$ (Ne X and S XV). The first two lines might contribute partially to the red wings of the observed features at 1223.6 and $1242.4 \AA$, while the third line is not visible in the current data. It should be noted that the third line might have a larger uncertainty than the first two since the Ne X emission is highly model-dependent (Fig. 6). The other absorbers reported in Baum et al. (2005) have velocity offsets in the range of $-1407 \mathrm{~km} \mathrm{~s}^{-1}$ to $533 \mathrm{~km} \mathrm{~s}^{-1}$, and their possible CX emission lines would either overlap with the absorption dips (H I) or shift out of the observed energy band ( Ne X and S XV). Hence it is not surprising that only one or few absorbers can be identified as candidate CX emitters with the current data. 


\subsection{CX lines expected at other wavelengths}

The current Hubble data can only provide a hint of the possible CX process; further observations covering a broader energy range are required to verify this scenario. Here we discuss the expected CX emission at X-ray and EUV wavelengths based on the CX model presented in Sect. 3. Assuming that the projectile ions have an ionization state of $\sim 1 \mathrm{keV}$ and a collision velocity of $500 \mathrm{~km} \mathrm{~s}^{-1}$, the brightest CX line of the related ions in the X-ray band would be the S XV $\mathrm{He} \alpha$ forbidden line at a rest-frame energy of $2.43 \mathrm{keV}$. Further assuming that the $\mathrm{S} \mathrm{XV}$ feature at $1242.4 \AA$ has a flux of $0.7 \times 10^{-15} \mathrm{erg} \mathrm{s}^{-1} \mathrm{~cm}^{-2}$ (Sect. 3), the flux of the He $\alpha \mathrm{CX}$ line is expected to be about $1.1 \times$ $10^{-14} \mathrm{erg} \mathrm{s}^{-1} \mathrm{~cm}^{-2}$, which is an order of magnitude lower than the thermal emission of the same line based on the CIE modelling of the Perseus ICM reported in Hitomi Collaboration et al. (2016a). The strongest Ne X CX line would be the Ly $\alpha$ line at the rest-frame energy of $1.02 \mathrm{keV}$, which is about $2 \%$ of the CIE line flux. The characteristic $\mathrm{SXV} n=9$ to $n=1$ and $\mathrm{NeX} n=6$ to $n=1$ transitions would appear at 3.19 and $1.32 \mathrm{keV}$, with fluxes of $1.8 \times 10^{-15} \mathrm{erg} \mathrm{s}^{-1} \mathrm{~cm}^{-2}$ and $2.2 \times 10^{-16} \mathrm{erg} \mathrm{s}^{-1} \mathrm{~cm}^{-2}$, respectively.

The brightest CX line in the EUV range is expected to be

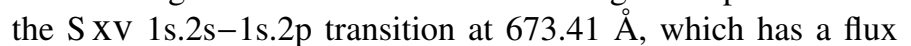
of $8.2 \times 10^{-15} \mathrm{erg} \mathrm{s}^{-1} \mathrm{~cm}^{-2}$. For NeX, the $3 \mathrm{~d}-4 \mathrm{f}$ transition at $187.42 \AA$ has a flux of $1.2 \times 10^{-15} \mathrm{erg} \mathrm{s}^{-1} \mathrm{~cm}^{-2}$.

Assuming solar abundances, we further calculate the CX emission for the other elements. As a notable ion, O VIII would have a bright CX Ly $\alpha$ of $6.6 \times 10^{-14} \mathrm{erg} \mathrm{s}^{-1} \mathrm{~cm}^{-2}$, which is more than one order of magnitude lower than the CIE Ly $\alpha$ emission based on the Hitomi Collaboration et al. (2016a) model. The flux of the $n=5$ to $n=1$ transition at $0.84 \mathrm{keV}$ is expected to be $3.6 \times 10^{-14} \mathrm{erg} \mathrm{s}^{-1} \mathrm{~cm}^{-2}$. Related to the wellknown $3.5 \mathrm{keV}$ line issue (Gu et al. 2015), this CX component might emit a SXVI $n=9$ to $n=1$ transition at a rest-frame energy of $3.45 \mathrm{keV}$, which would have a flux of $5.5 \times 10^{-16} \mathrm{erg} \mathrm{s}^{-1} \mathrm{~cm}^{-2}$. This value is substantially lower than the observed S XVI CX line with the Hitomi telescope $(\sim 4 \times$ $10^{-14} \mathrm{erg} \mathrm{s}^{-1} \mathrm{~cm}^{-2}$; Hitomi Collaboration et al. 2016b), suggesting that other $\mathrm{CX}$ components might also exist in the Perseus core.

Despite the low fluxes in X-rays, the expected CX emission might still be detectable, since the CX lines would probably be shifted significantly from the CIE lines of the Perseus cluster. The current Hitomi data is not suitable for this study, due to the lack of collecting area below $\sim 3 \mathrm{keV}$ (Hitomi Collaboration et al. 2016a). In the future, the high-resolution X-ray spectrometers on board the X-ray astronomy recovery mission (XARM) and Athena will greatly improve our understanding of the CX phenomenon in this and other extragalactic objects.

\section{Summary}

Based on a newly developed CX emission model, we demonstrate that the CX process between hot and cold cosmic plasma is naturally more efficient in creating UV emission than the thermal processes. By reanalysing the Hubble/STIS spectrum of the core of NGC 1275, we detect three possible UV line features at $1223.6 \AA, 1242.4 \AA$, and $1244.0 \AA$, which can be simultaneously explained by $\mathrm{CX}$ reactions between highly ionized hydrogen, neon, and sulfur with atomic hydrogen. Our model shows that the CX emitter has a line-of-sight velocity offset of $\sim-3400 \mathrm{~km} \mathrm{~s}^{-1}$ with respect to NGC 1275 , which is roughly consistent with one of the $\operatorname{Ly} \alpha$ absorption structures. Hence we speculate that the possible CX emitter and the absorber might share the same origin, the outflowing clouds in the vicinity of the nucleus.

Acknowledgements. SRON is supported financially by NWO, The Netherlands Organization for Scientific Research.

\section{References}

Baum, S. A., Laor, A., O’Dea, C. P., Mack, J., \& Koekemoer, A. M. 2005, ApJ, 632, 122

Belkić, D. 1991, Phys. Scr., 43, 561

Belkić, D., Gayet, R., \& Salin, A. 1992, At. Data Nucl. Data Tables, 51, 59

Branduardi-Raymont, G., Bhardwaj, A., Elsner, R. F., et al. 2007, A\&A, 463, 761

Chevalier, R. A., Kirshner, R. P., \& Raymond, J. C. 1980, ApJ, 235, 186

Conselice, C. J., Gallagher, III, J. S., \& Wyse, R. F. G. 2001, AJ, 122, 2281

Cravens, T. E. 1997, Geophys. Res. Lett., 24, 105

Cumbee, R. S., Liu, L., Lyons, D., et al. 2016, MNRAS, 458, 3554

Dennerl, K. 2010, Space Sci. Rev., 157, 57

Dennerl, K., Lisse, C. M., Bhardwaj, A., et al. 2006, A\&A, 451, 709

Erickson, G. W. 1977, J. Phys. Chem. Ref. Data, 6, 831

Fabian, A. C., Sanders, J. S., Williams, R. J. R., et al. 2011, MNRAS, 417, 172

Fujimoto, R., Mitsuda, K., Mccammon, D., et al. 2007, PASJ, 59, 133

Gross, E., \& Vitells, O. 2010, Eur. Phys. J. C, 70, 525

Gu, M. F. 2008, Can. J. Phys., 86, 675

Gu, L., Yagi, M., Nakazawa, K., et al. 2013, ApJ, 777, L36

Gu, L., Kaastra, J., Raassen, A. J. J., et al. 2015, A\&A, 584, L11

Gu, L., Kaastra, J., \& Raassen, A. J. J. 2016a, A\&A, 588, A52

Gu, L., Mao, J., Costantini, E., \& Kaastra, J. 2016b, A\&A, 594, A78

Harel, C., Jouin, H., \& Pons, B. 1998, At. Data Nucl. Data Tables, 68, 279

Hitomi Collaboration, Aharonian, F., Akamatsu, H., et al. 2016a, Nature, 535, 117

Hitomi Collaboration, Aharonian, F. A., Akamatsu, H., et al. 2016b, ApJ, 837, L15

Katsuda, S., Tsunemi, H., Mori, K., et al. 2011, ApJ, 730, 24

Kraemer, S. B., Crenshaw, D. M., \& Gabel, J. R. 2001a, ApJ, 557, 30

Kraemer, S. B., Crenshaw, D. M., Hutchings, J. B., et al. 2001b, ApJ, 551, 671

Kramida, A. E. 2010, At. Data Nucl. Data Tables, 96, 586

Kramida, A., Ralchenko, Yu., Reader, J., \& NIST ASD Team. 2015, NIST

Atomic Spectra Database (ver. 5.3), http://physics.nist.gov/asd

[2016, November 30]. National Institute of Standards and Technology, Gaithersburg, MD

Krasnopolsky, V. A., \& Mumma, M. J. 2001, ApJ, 549, 629

Krasnopolsky, V. A., Greenwood, J. B., \& Stancil, P. C. 2004, Space Sci. Rev., 113,271

Kriss, G. A., Green, R. F., Brotherton, M., et al. 2000, ApJ, 538, L17

Kriss, G. A., Arav, N., Kaastra, J. S., et al. 2011, A\&A, 534, A41

Lallement, R. 2009, Space Sci. Rev., 143, 427

Lisse, C. M., Dennerl, K., Englhauser, J., et al. 1996, Science, 274, 205

Liu, J., Mao, S., \& Wang, Q. D. 2011, MNRAS, 415, L64

Liu, L., Wang, J. G., \& Janev, R. K. 2014, Phys. Rev. A, 89, 012710

Lodders, K., \& Palme, H. 2009, Meteoritics and Planetary Science Supplement, 72,5154

Martin, W. C., Zalubas, R., \& Musgrove, A. 1990, J. Phys. Chem. Ref. Data, 19, 821

Mehdipour, M., Kaastra, J. S., \& Kallman, T. 2016, A\&A, 596, A65

Mullen, P. D., Cumbee, R. S., Lyons, D., \& Stancil, P. C. 2016, ApJS, 224, 31

Pequignot, D., Stasińska, G., \& Aldrovandi, S. M. V. 1978, A\&A, 63, 313

Pinto, C., Fabian, A. C., Ogorzalek, A., et al. 2016, MNRAS, 461, 2077

Pollock, A. M. T. 2007, A\&A, 463, 1111

Schaal, K., Springel, V., Pakmor, R., et al. 2016, MNRAS, 461, 4441

Scharwächter, J., McGregor, P. J., Dopita, M. A., \& Beck, T. L. 2013, MNRAS, 429, 2315

Shah, C., Dobrodey, S., Bernitt, S., et al. 2016, ApJ, 833, 52

Shull, J. M., Stevans, M., \& Danforth, C. W. 2012, ApJ, 752, 162

Smith, R. K., Foster, A. R., Edgar, R. J., \& Brickhouse, N. S. 2014, ApJ, 787, 77 Snowden, S. L., Collier, M. R., \& Kuntz, K. D. 2004, ApJ, 610, 1182

Stevans, M. L., Shull, J. M., Danforth, C. W., \& Tilton, E. M. 2014, ApJ, 794, 75

Tsuru, T. G., Ozawa, M., Hyodo, Y., et al. 2007, PASJ, 59, 269

Urdampilleta, I., et al. 2017, A\&A, submitted

Vazza, F., Dolag, K., Ryu, D., et al. 2011, MNRAS, 418, 960

Walker, S. A., Kosec, P., Fabian, A. C., \& Sanders, J. S. 2015, MNRAS, 453, 2480 